\title{
Managing Opioid-Tolerant Patients in the Perioperative Surgical Home.
}

\author{
John T. Wenzel \\ Thomas Jefferson University \\ Eric S. Schwenk \\ Thomas Jefferson University \\ Jaime L. Baratta \\ Thomas Jefferson University \\ Eugene R. Viscusi \\ Thomas Jefferson University
}

Follow this and additional works at: https://jdc.jefferson.edu/anfp

Part of the Anesthesiology Commons

Let us know how access to this document benefits you

\section{Recommended Citation}

Wenzel, John T.; Schwenk, Eric S.; Baratta, Jaime L.; and Viscusi, Eugene R., "Managing OpioidTolerant Patients in the Perioperative Surgical Home." (2016). Department of Anesthesiology Faculty Papers. Paper 29.

https://jdc.jefferson.edu/anfp/29

This Article is brought to you for free and open access by the Jefferson Digital Commons. The Jefferson Digital Commons is a service of Thomas Jefferson University's Center for Teaching and Learning (CTL). The Commons is a showcase for Jefferson books and journals, peer-reviewed scholarly publications, unique historical collections from the University archives, and teaching tools. The Jefferson Digital Commons allows researchers and interested readers anywhere in the world to learn about and keep up to date with Jefferson scholarship. This article has been accepted for inclusion in Department of Anesthesiology Faculty Papers by an authorized administrator of the Jefferson Digital Commons. For more information, please contact: JeffersonDigitalCommons@jefferson.edu. 


\section{Managing the Opioid-Tolerant Patient in the Perioperative Surgical Home}

Corresponding author:

John T. Wenzel, MD

Sidney Kimmel Medical College, Thomas Jefferson University

Department of Anesthesiology

Suite 8130, Gibbon Building

111 South $11^{\text {th }}$ Street

Philadelphia, PA 19107

Phone: 215-955-6161

Fax: 215-955-0677

Email: John.Wenzel@jefferson.edu

Co-authors:

Eric S. Schwenk, MD

Sidney Kimmel Medical College, Thomas Jefferson University

Department of Anesthesiology

Suite 8130, Gibbon Building

111 South $11^{\text {th }}$ Street

Philadelphia, PA 19107

Phone: 215-955-6161

Fax: 215-955-0677

Email: Eric.Schwenk@jefferson.edu

Jaime L. Baratta, MD

Sidney Kimmel Medical College, Thomas Jefferson University

Department of Anesthesiology

Suite 8130, Gibbon Building

111 South $11^{\text {th }}$ Street

Philadelphia, PA 19107

Phone: 215-955-6161

Fax: 215-955-0677

Email: Jaime.Baratta@jefferson.edu

Eugene R. Viscusi, MD

Sidney Kimmel Medical College, Thomas Jefferson University

Department of Anesthesiology

Suite 8490, Gibbon Building

111 South $11^{\text {th }}$ Street

Philadelphia, PA 19107

Phone: 215-955-6161

Fax: 215-955-0677

Email: Eugene.Viscusi@jefferson.edu 


\section{$\underline{\text { Disclosures: }}$}

John Wenzel has no financial disclosures.

Eric Schwenk has no financial disclosures.

Jaime Baratta has no financial disclosures.

Eugene Viscusi: Research grants to my institution - AcelRx, Cumberland, Pacira

Consulting/honoraria - AcelRx, Malinckrodt, The Medicines Company, Merck, Salix, Pacira, Trevena

Key Words: opioid tolerance, multimodal analgesia, perioperative surgical home, buprenorphine

\section{$\underline{\text { Key Points: }}$}

- In the perioperative surgical home model, anesthesiologists are well positioned to manage complex patients, including those who are opioid-tolerant, due to their training and expertise in pharmacology.

- Opioid-tolerant patients present challenges for postoperative analgesia, posing dual risks of poor pain control and medication-related toxicity.

- Reduction of opioids through regional anesthesia techniques and multimodal non-opioid agents can improve analgesia and minimize opioid-related complications in the high-risk opioid-tolerant population.

Synopsis:

Management of acute postoperative pain is important to decrease perioperative morbidity and improve patient satisfaction with the healthcare experience. Although they are indispensable agents in most surgical settings, opioids are associated with potential adverse events that may lead 
to significant risk. Uncontrolled pain is a risk factor in the transformation of acute pain to chronic pain. Balancing these issues can be especially challenging in the opioid-tolerant patient undergoing surgery, for whom rapidly escalating opioid doses in an effort to control pain can be associated with increased complications. In the perioperative surgical home model, anesthesiologists are positioned to coordinate a comprehensive perioperative analgesic plan that begins with the preoperative assessment and continues through discharge. Multimodal analgesia may improve pain control while reducing opioid-related complications, and should include regional techniques when possible and adjunct agents throughout the perioperative period to reduce opioid requirements. With patient safety as a guiding principal, a physician-led acute pain service can coordinate the appropriate approaches to postoperative monitoring and the management of peripheral nerve catheters, parenteral drug delivery techniques, and conversion to oral opioid regimens. 
$\underline{\text { Introduction }}$

Acute pain is a major concern of many patients preparing to undergo surgery. The incidence of postoperative acute pain varies widely in the literature. It has been reported as high as $80 \%$ and is likely underreported (1). Such data suggest that uncontrolled acute postoperative pain continues to be an unmet need and a target for improvement. This is further compounded in the opioid-tolerant patient receiving chronic opioids at baseline. While preparing to manage the opioid-tolerant patient, mechanisms underlying chronic postsurgical pain, acute opioid tolerance, and opioid-induced hyperalgesia are poorly understood but highly relevant. The emerging model of the perioperative surgical home puts anesthesiologists in position to best address complicated patients, particularly those with pre-existing chronic pain conditions and who are opioid-tolerant as a result of chronic opioid therapy.

\section{The Perioperative Surgical Home}

The perioperative surgical home (PSH) has been supported by the American Society of Anesthesiologists (ASA) to improve outcomes while improving efficiency, broadening the role of the anesthesiologist in preoperative, intraoperative, and postoperative care. It is defined by the ASA as "a patient-centered and physician-led multidisciplinary and team-based system of coordinated care that guides the patient throughout the entire surgical experience" (2). The "Triple Aim" goals of the PSH as described by Berwick et al (3) include: 1) improving the individual experience of care, 2) improving the health of populations, and 3) reducing the per capita cost of care.

The PSH for surgical patients has been compared to the Patient-Centered Medical Home (PCMH) for primary care. Recent data suggest that the PCMH improves outcomes and reduces 
costs (4). Development of the PSH requires the support of hospital administration and collaboration of surgeons and anesthesiologists. Anesthesiologists are particularly well suited to staff the PSH (4) as the physician team leader. Since many institutions do not have an existing preoperative anesthesia clinic, development of a PSH can be financially challenging. If there is institutional support or desire for PSH development, the institution and anesthesia practice need to be financially "aligned" with an agreement to support funding (4). Compensation for staffing also needs to be considered. Hospital administration may be willing to compensate anesthesiologists for this practice if a financial benefit can be demonstrated. Reimbursement for anesthesiologists may mirror that of internists in the PCMH, as the Centers for Medicare and Medicaid Services have recognized the value of the PCMH (Kain). Future payment models that include bundled payments for services may make anesthesiologist compensation complicated.

Once the PSH is implemented, services begin with early patient engagement after the decision for surgery is made. The surgical experience is treated as a fluid continuum rather than discrete pre-, intra-, and post-surgical phases. It involves appropriate risk stratification and preoperative testing, decreased redundancy in testing, improved operating room efficiency, decreased variability through the use of evidence-based surgical care pathways, and postsurgical care initiatives (5). We will focus on management of the opioid-tolerant patient within the PSH.

\section{Opioids}

Opioids remain a mainstay of analgesia regimens for the surgical patient. Prescriptions for opioids, as well as the increased incidence of prescription opioid abuse have been on the rise in recent years, especially in the United States and Canada. Opioid-related deaths and adverse events have seen a similar spike (6). Yet, despite the increased use of opioid medications to manage pain 
and improve function, there remains a serious and significant mismatch between the number of prescriptions issued and positive end-outcomes of pain care in the United States (7). In some patients, chronic opioid exposure, especially at high doses, has been attributed to to a progressive reduction in analgesia while increasing risks of opioid tolerance, opioid-induced hyperalgesia, and medication misuse (8). These issues come into focus when patients on chronic opioid therapy have indications for surgery.

\section{Opioid Tolerance}

- A phenomenon whereby increasing opioid doses are required for analgesia due to a desensitization of pain signaling at the opioid receptor (9).

- Acute opioid tolerance (AOT) may develop rapidly in patients who are opioid-naïve (10).

- Patients exposed to high intraoperative opioid doses have demonstrated increased postoperative pain and opioid requirements.

- These higher opioid doses are associated with an increased risk of adverse events (9).

\section{Opioid-Induced Hyperalgesia $(\mathrm{OIH})$}

- Nociceptive sensitization likely caused by neuroplastic changes in the central and peripheral nervous systems caused by opioid exposure, leading to paradoxical worsening of pain with increasing opioid doses.

- Remifentanil has been particularly implicated, but it may occur with acute or chronic exposure, high or low doses, and any opioid or route of administration (11).

- Prevalence of OIH is likely underappreciated as its diagnosis can be very challenging and it can be confused with tolerance, worsening disease, or even opioid withdrawal. 
- When $\mathrm{OIH}$ is suspected, opioid dose reduction may improve analgesia. Other proposed treatments include opioid rotation, $N$-methyl-D-aspartate (NMDA) receptor antagonists (ketamine, memantine, dextromethorphan), interventional pain management, or behavioral management (11).

- Rotation to methadone is of particular interest since it is both a mu-receptor opioid agonist and an NMDA-receptor antagonist.

Managing acute postoperative pain may be especially challenging in the opioid-tolerant patient, and increasing doses of opioids to overcome tolerance is a poor management strategy as this may result in unwanted opioid side effects, including sedation, respiratory depression, ileus, and paradoxical worsening of pain. Multimodal analgesia involves the incorporation of several medications with unique mechanisms of action in an effort to improve analgesia and minimize the side effects of any one class of medication, especially opioids. These patients may benefit the most from preoperative optimization of their pain regimen, context-appropriate selection of intraoperative anesthetic techniques, and a multimodal postoperative analgesic plan.

\section{$\underline{\text { Preoperative Assessment }}$}

In the $\mathrm{PSH}$, the preoperative assessment begins in the preoperative clinic rather than at the bedside on the day of surgery. Patients often encounter their anesthesiologist for the first time in the pre-surgery "holding area" minutes before surgery, whereas a previously scheduled comprehensive assessment in the clinic affords time to enact a management plan for the opioidtolerant patient. This anticipatory planning and care coordination also creates the opportunity to provide perioperative education, to set expectations, and alleviate patient anxiety. Managing 
patient expectations for pain management is critical to success, and enhances patient satisfaction. Both surgeon and anesthesiologist should be attuned to early identification and management of the opioid-tolerant patient.

Assessment begins with a thorough history and physical examination and includes elucidation of the location and nature of chronic pain, as well the degree of functional impairment. Particular attention should be paid to the patient's current pain regimen, including exact doses, schedule, route(s) of administration, prescriber, and effects (positive and negative). A complete history includes past medication use and experiences (Table 1). Patients who cannot provide evidence of accountability for their opioids justify additional exploration into opioid abuse, misuse or diversion. A urine drug screen may be useful to determine potential substance misuse, abuse or diversion. In the face of active, undiagnosed substance abuse, a joint decision between the surgeon and anesthesiologist should be made, weighing the risks of the abuse on both the patient's current health and surgical outcome. A more detailed discussion of substance abuse is out of the scope of this article.

The nature of the proposed surgery is important in determining the anesthetic and analgesic plan. Is it major surgery requiring hospital admission with high levels of anticipated pain? On the other hand, is discharge home on the same day anticipated? Is the procedure and medical circumstances of the patient suitable for a regional anesthetic technique? Would any drugs or techniques be contraindicated because of patient characteristics (e.g. renal or liver disease) or surgical type (e.g. positioning, bleeding risk or bone healing)?

Taking into account the patient's medical history, planned surgery, current therapies, and existing and anticipated pain, the anesthesiologist may tailor a specific anesthetic plan that involves a combination of pre-emptive analgesia, regional techniques, intraoperative agents, and 
postoperative multimodal analgesia. Multimodal regimens are especially important for the opioidtolerant patient, with the goals of adequate analgesia, prevention of chronic post-surgical pain (CPSP), and minimization of opioids and their associated adverse events. Chronic exposure to opioids reduces drug efficacy, requiring increasing doses to achieve similar analgesic effects. These patients may be at increased risk when compared to opioid-naïve patients, where increasing dosages to improve efficacy increases risk of adverse events, most significantly respiratory depression. Additionally, opioid-tolerant patients presenting for surgery may have altered pain sensitivity that includes generalized hyperalgesia or ill-defined pain remote to the surgical site (12). We are therefore left to maximize the utility of non-opioid agents and regional techniques.

The advantages of a patient-specific analgesic plan are several-fold: first, the patient will be well informed about expectations and will not be expected to make decisions about his or her care immediately prior to or after surgery when they are likely to be in a more anxious, distressed, or cognitively impaired state from sedatives or anesthetics. From an ethical standpoint, this is important with regard to adequacy of informed consent. Second, communication is improved with the intraoperative anesthesia team when there is sufficient time for concerns of all parties to be elicited, discussed and clarified. Finally, this early encounter and assessment affords time to prepare for and enact management plans for specific therapies. 
Table 1: Components of the preoperative assessment for the opioid-tolerant patient.

\section{The Preoperative Assessment}

- Clarification of pre-existing pain and functional limitations

- Current opioid medications, including dose, frequency, and any changes in dose in the past six months

- Current opioid prescriber and dispensing pharmacy

- Current non-opioid analgesics (e.g. acetaminophen, NSAIDs, COX-2 inhibitors, antineuropathic agents, benzodiazepines, muscle relaxants)

- Past opioid medications and their effectiveness and tolerability of side effect profile

- Review of potential misuse or abuse of opioids

- Discussion of expectations and anticipated level of pain associated with the particular procedure (mild, moderate, severe) including perceptions from past surgical experiences 


\section{Opioid Agonists}

The anesthesiologist must first determine which patients require extra attention and should be considered "opioid-tolerant." Tolerance is defined by the FDA as the use of greater than or equal to $60 \mathrm{mg}$ of oral morphine equivalents per day for 7 days or longer, regardless of long-term opioid use (5). Opioid-tolerant patients may be taking a variety of opioids at home in immediaterelease, short-acting (e.g. oxycodone, hydrocodone, morphine, oral transmucosal fentanyl) or extended-release, long-acting (e.g. MSContin, OxyContin, transdermal fentanyl patch) preparations. Routes of administration may include oral, subcutaneous, transdermal, or intrathecal delivery. Patients should be advised to continue their usual analgesic regimen, including regularly scheduled immediate- or extended-release and transdermal agents.

Methadone is a mu-receptor opioid agonist that may be prescribed for the treatment of chronic pain or as a once-daily opioid replacement therapy for addiction. These patients have tolerance to opioids, but may also have increased pain sensitivity (13). Patients on methadone for both chronic pain or methadone maintenance therapy (MMT) should continue their scheduled regimen perioperatively to avoid fluctuations in methadone levels given its long half-life $(13,14)$.

An evidence-based multimodal analgesic plan is necessary for opioid-tolerant patients to provide adequate analgesia and prevent withdrawal but also to reduce the length of hospital stay and prevent occurrences of readmission (5). Despite the FDA definition of tolerance, in clinical acute pain practice, chronic use of relatively low levels of daily opioid exposure (20 mg morphine equivalents daily for greater than 7-10 days) can significantly reduce the effectiveness of postoperative opioids, leading to poorly controlled pain.

The specific components of any multimodal analgesic plan will vary based on specific patient and surgical factors, but includes regional or neuraxial anesthesia/analgesia when possible, 
non-opioid adjunct agents (e.g. NSAIDs/COX-2 inhibitors, acetaminophen, gabapentinoids, alpha-agonists, intravenous lidocaine), and NMDA-receptor antagonists (e.g. ketamine), using opioids for rescue analgesia only. Preoperative dosing of non-opioid analgesics is gaining popularity and typically involves medications taken prior to surgery and continued around-theclock postoperatively (Table 2). Pregabalin $(15,16,17,18)$, gabapentin, and celecoxib (19) have demonstrated benefit when started preoperatively in reducing the need for postoperative opioids. The combination of acetaminophen, pregabalin, and celecoxib has shown benefit when started preoperatively and continued around-the-clock postoperatively (20). At the authors' institution, this has been adopted for orthopedic, urologic, and gynecologic procedures. 
Table 2. Multimodal Non-opioid Agents for Preemptive Analgesia

\begin{tabular}{|l|l|l|}
\hline Drug & $\begin{array}{l}\text { Administration } \\
\text { Route }\end{array}$ & Suggested Dose (Two hours before surgery) \\
\hline Acetaminophen & PO & $1000 \mathrm{mg}($ over $50 \mathrm{~kg})$ \\
\hline Celecoxib & PO & $200-400 \mathrm{mg}$ \\
\hline Pregabalin & PO & $75-150 \mathrm{mg}$ \\
\hline Gabapentin & PO & $900-1200 \mathrm{mg}$ \\
\hline
\end{tabular}




\section{$\underline{\text { Intraoperative Management }}$}

In the PSH model, the anesthesiologist caring for the patient on the day of surgery will have the advantage of the thorough preoperative assessment and management strategy already in place. The anesthesiologist can then advise the patient of the finalized anesthetic plan. Patients should continue their analgesic regimen up until the time of surgery, including any morning doses of opioid.

While tailoring the analgesic plan, the anesthesiologist must also take into account the nature of a patient's pain and the mechanisms of analgesic agents to be used. Pain is complex and subjective and there are five generally recognized categories of pain: nociceptive, neuropathic, psychogenic, mixed and idiopathic (21):

- Nociceptive pain can be described as sharp or dull, aching, throbbing, pressure-like or feelings of stiffness.

- Neuropathic pain is often burning, shock-like tingling or stabbing.

- Mixed pain is a result of stimulation of both nociceptive and neuropathic pathways.

- Psychogenic pain is pain that is thought to be an emotional or psychiatric phenomenon, only to be considered once all other nociceptive or neuropathic etiologies have been ruled out.

- Idiopathic pain is due to unknown origin.

The concept of multimodal analgesia takes into account understanding the type of pain in need of treatment and targeting it at multiple sites along the pain pathway to achieve a synergistic effect with different classes of analgesics.

- Opioids act both supraspinally via inhibitory pathways as well as in the dorsal horn to impede nociception. 
- NSAIDs act both in the spinal cord and periphery, inhibiting cyclooxygenase to decrease inflammation secondary to prostaglandins and further decrease nociception.

- Acetaminophen acts centrally likely via inhibition of cyclooxygenase in treating nociceptive pain.

- Local anesthetics act at central, spinal and peripheral sites, depending on route or localization of administration.

- NMDA receptor antagonists, exerting effects in the dorsal horn, inhibit nociceptive and neuropathic pain.

- Gabapentinoids bind to calcium channels in the spinal cord and brain to decrease neuropathic pain.

Although opioid-tolerant patients presenting for surgery will need opioids perioperatively, multimodal pain regimens are paramount in managing acute surgical pain in these cases. This may include the use of intraoperative adjuncts such as epidural/ regional analgesia, ketamine, dexmedetomidine, and lidocaine infusions.

\section{Ketamine}

A single dose of opioid will lead to activation of NMDA receptors (22). In theory, tolerance and hyperalgesia begins with the first dose of an opioid. In animal studies, ketamine, an NMDAreceptor antagonist, reversed morphine tolerance and improved effectiveness while additionally preventing acute tolerance. (23). The use of ketamine as an analgesic adjunct in opioid-naïve patients resulted in decreased opioid requirements after surgery, fewer opioid-related side effects such as nausea and vomiting, and possibly prevention of chronic pain and hyperalgesia $(24,25$, 
26). Few studies, however, have examined the effect of intraoperative ketamine in opioid-tolerant patients.

A study by Loftus et al (27) showed that intraoperative ketamine infusions resulted in reduced opioid requirements in the first 48 hours and at 6 weeks postoperatively in opioiddependent patients undergoing back surgery. Additionally, average pain scores were significantly reduced in the postanesthesia care unit and at 6 weeks postoperatively (27). The authors recommended patients taking greater than or equal to $30 \mathrm{mg}$ per day of morphine equivalents with expected moderate-severe postsurgical pain receive a bolus of ketamine of $0.5 \mathrm{mg} / \mathrm{kg}$ followed by infusion of $0.25 \mathrm{mg} / \mathrm{kg} / \mathrm{hr}$ intraoperatively.

Postoperatively, ketamine infusions may be safely administered without continuous cardiac or respiratory monitoring (ICU or telemetry) (28). An in-house APMS team is crucial to provide regular monitoring of analgesia and titration. Although studies demonstrate that it is well tolerated, patients should be monitored for neurological and psychological side effects.

\section{Regional Anesthesia and Analgesia}

A number of surgical procedures, including extremity surgery, carotid endarterectomy, and hernia repair, may be done under a regional/neuraxial block as the sole anesthetic, thus decreasing the reliance of opioids in an opioid-tolerant patient.

Although general anesthesia is often indicated, regional analgesia as part of a multimodal regimen decreases opioid burden in these challenging patients. The use of long-acting local anesthetics and continuous catheters enable regional analgesia to be effective during the first few days following surgery when acute pain is most severe. 
Utilization of perineural catheters results in prolonged postoperative pain control (including resting and dynamic pain), accelerated resumption of therapy, less reliance on opioids, fewer sleep disturbances, and overall increased patient satisfaction (29). Epidural analgesia results in improved rehabilitation, better pain control, and decreased opioid consumption when compared to opioids alone (30).

Appropriate regional techniques, including single-shot or continuous peripheral nerve blocks, or epidural analgesia, should be employed whenever possible. Although regional anesthesia offers many benefits in opioid-tolerant patients, opioids must judiciously be continued to prevent withdrawal and to treat the chronic pain not related to the surgical procedure.

\section{Lidocaine Infusions}

The use of an intravenous lidocaine infusion has been widely studied in abdominal surgery. In open and laparoscopic abdominal surgery, an intravenous lidocaine infusion resulted in decreased postoperative pain for 48 hours postoperatively, reduced opioid consumption, earlier return of bowel function, earlier rehabilitation and decreased length of stay (31).

When compared to thoracic epidural analgesia in patients undergoing colorectal surgery, intraoperative and postoperative lidocaine infusions resulted in similar times to return of bowel function and time to discharge; however, epidural analgesia resulted in lower numeric pain scores (32).

The benefit of intraoperative lidocaine infusions may not apply to all types of surgery as the addition of lidocaine in patients undergoing mastectomy did not result in decreased pain or reduced opioid consumption when compared to placebo (33). Although the use of intraoperative lidocaine infusions has not been well studied in the opioid-tolerant population, current literature 
suggests it may be useful as part of a multimodal regimen when regional techniques are contraindicated. A common dosing strategy is $1.5 \mathrm{mg} / \mathrm{kg}$ loading dose followed by $1.5 \mathrm{mg} / \mathrm{kg} / \mathrm{hr}$ intraoperatively.

\section{Dexmedetomidine}

With high density of receptors in the dorsal horn, alpha- 2 adrenergic agonists act on the central nervous system to reduce pain. Dexmedetomidine is a highly selective alpha-2 agonist that acts as a sedative, anxiolytic, sympatholytic, and analgesic with minimal respiratory depression.

In rats with presumed neuropathic pain, dexmedetomidine exhibited anti-hyperalgesic action (34). In patients undergoing laparoscopic bariatric surgery, randomized to receive $0.2,0.4$ or $0.8 \mathrm{mcg} / \mathrm{kg} / \mathrm{h}$ dexmedetomidine infusion intraoperatively, dexmedetomidine decreased opioid use, need for antiemetic treatment, and length of stay in the PACU, but did not change opioid use patterns or quality of recovery beyond the PACU (35).

Given the cardiovascular effects of dexmedetomidine infusions, namely bradycardia and hypotension, Tufanogullari et al (35) recommended $0.2 \mathrm{mcg} / \mathrm{kg} / \mathrm{h}$ infusion intraoperatively as an analgesic adjunct. Gurbet et al (36) showed an intraoperative dexmedetomidine rate of 0.5 $\mathrm{mcg} / \mathrm{kg} / \mathrm{hr}$ decreased 48 -hour opioid consumption with no change in pain scores following total abdominal hysterectomy when compared to placebo. Although dexmedetomidine has not been specifically studied in the opioid-tolerant population, the literature suggests a continuous infusion during surgery may decrease intraoperative opioid requirements. It is likely that continuation of dexmedetomidine is necessary to extend opioid reduction beyond the PACU.

\section{Postoperative Management}


The care of the opioid-tolerant patients continues well beyond the operating room and into the post-anesthesia care unit (PACU) as part of the PSH model. Although the surgeon typically orders analgesics for the patients when on the medical ward, there is substantial opportunity for input and collaboration by the anesthesiologist. Medication management and expectations for postoperative analgesia can be established early on and can help create a positive patient experience.

In this section, strategies to managing postoperative analgesia for the opioid-tolerant patient will be discussed, including:

- Benefits of a non-opioid multimodal approach

- Resuming home medications, including opioid agonist-antagonists

- The role of acute pain services in the perioperative surgical home model

\section{Multimodal Approach to Postoperative Analgesia}

Inadequate postoperative pain management has been linked with complications, including myocardial ischemia, impaired pulmonary function, ileus, thromboembolism, impaired immune function, wound infection and anxiety (37). Additionally, hospital reimbursements are now linked to patient satisfaction scores (HCAHPS). Pain is a significant driver of poor patient satisfaction. Poor outcomes related to uncontrolled pain result in greater healthcare costs.

Multimodal analgesia has been successfully implemented into multiple surgical pathways, and has been shown to reduce opioid consumption (38), surgical complications $(38,39)$, and length of hospital stay $(38,39,40)$. Some agents that have been used successfully in multimodal pathways include acetaminophen (PO and IV), cyclooxygenase-2 (COX-2) inhibitors, gabapentinoids (gabapentin and pregabalin), non-steroidal anti-inflammatory drugs (NSAIDs), glucocorticoids, 
local anesthetics, $\alpha-2$ agonists (clonidine and dexmedetomidine), and ketamine (Table 3 ). To the extent possible, these agents can be used to minimize opioids and improve analgesia. 
Table 3. Multimodal Non-Opioid Agents for Postoperative Analgesia

\begin{tabular}{|l|l|l|}
\hline Drug & $\begin{array}{l}\text { Administration } \\
\text { Route }\end{array}$ & Suggested Dose and Frequency \\
\hline Acetaminophen & PO/IV & $1000 \mathrm{mg} \mathrm{q6h}($ over $50 \mathrm{~kg})$ \\
\hline Ibuprofen & PO & $600 \mathrm{mg} \mathrm{q} 6 \mathrm{~h}$ \\
\hline Ketorolac & PO/IV & $15-30 \mathrm{mg} \mathrm{q} 6 \mathrm{~h}$ \\
\hline Celecoxib & PO & $200-400 \mathrm{mg} \mathrm{q} 12 \mathrm{~h}$ \\
\hline Gabapentin & PO & $600-800 \mathrm{mg}$ TID \\
\hline Pregabalin & PO & $75-150 \mathrm{mg} \mathrm{q} 12 \mathrm{~h}$ \\
\hline Ketamine & IV & $0.5 \mathrm{mg} / \mathrm{kg}$ bolus; infusion $0.25 \mathrm{mg} / \mathrm{kg} / \mathrm{hr}$ \\
\hline
\end{tabular}


Multimodal analgesia pathways have existed for more than 15 years but Kehlet and colleagues (41) were the first to describe pathways leading to clinically important outcomes, such as reduction in length of hospital stay in colorectal procedures. There have been several large reviews and meta-analyses summarizing the literature on several multimodal agents, with both gabapentin (42) and pregabalin (43) demonstrating an opioid-reducing effect when used in a multimodal pathway. The addition of a NSAID to an opioid as part of a multimodal pathway provides better analgesia than the opioid alone (44). Taken together, these studies provide evidence that using non-opioid agents whenever possible can improve analgesia, and this is even more so for the opioid-tolerant patient.

Because the doses of opioids taken chronically by the opioid-tolerant population often exceed many physicians' comfort levels, surgeons frequently seek recommendations on discharge regimens. Conversion from IV to oral opioids requires experience and expertise of a physician experienced in pain management (45).

\section{Postoperative Oral Analgesics and Restarting Home Medications}

If the patient was taking a combination opioid preoperatively (e.g.

hydrocodone/acetaminophen), that creates an opportunity for the anesthesiologist to initiate a discussion with the surgeon about postoperative analgesics. Non-opioid adjuncts, such as acetaminophen, NSAIDs, and gabapentinoids, should be initiated as the mainstay of treatment, with opioids for breakthrough pain only. Unless the patient underwent gastrointestinal surgery or cannot take anything by mouth for other reasons, oral medications may be started on the evening of the day of surgery. However, for patients admitted to the hospital after painful surgery, intravenous opioids are often needed. Opioids delivered via IV patient-controlled analgesia (PCA) 
lead to better patient satisfaction than intermittent boluses given via oral, IM, or IV routes, as well as lower rates of adverse events (46). More potent opioids, such as hydromorphone or sufentanil, may be considered when treating opioid-tolerant patients. Higher-dose PCA settings may be needed to deliver adequate analgesia.

\section{Special Considerations: Opioid Addiction Therapy}

Patients treated for opioid addiction present a particular challenge in that many have a history of longstanding opioid abuse with significant tolerance and hyperalgesia. It is not unusual for patients in recovery to be exceedingly fearful of exposure to non-maintenance opioids if they are on such a regimen, or re-exposure to opioids if they have managed to be fully abstinent. In addition to the opioid agonist methadone, patients being treated with opioid-replacement therapy (ORT) may be maintained on buprenorphine (categorized as a partial agonist, although its pharmacology is debated) or an opioid antagonist (naltrexone), each with unique considerations.

\section{Buprenorphine}

Buprenorphine is a semisynthetic opioid with a high mu-receptor binding affinity. It is marketed as a sole agent (Subutex) or in combination with naloxone (Suboxone) to deter abuse. A transbuccal patch delivery form of buprenorphine has been recently approved by the FDA for the treatment of chronic pain, There are limited data to guide perioperative management of patients taking buprenorphine; furthermore, its high binding affinity, slow dissociation, and analgesic “ceiling effect" may make adequate analgesia a challenge. 
Analgesic strategies for patients on buprenorphine mainly stem from expert opinion and case reports. Bryson (13) describes two basic treatment options as continuing buprenorphine throughout the perioperative period or stopping buprenorphine prior to surgery.

There is no clear consensus on the best strategy for managing buprenorphine preoperatively, with many recommending it be stopped preoperatively to avoid the analgesic ceiling effect of buprenorphine or the high dose of supplemental opioids that may be required. Discontinuation of ORT, however, carries a risk of relapse that will need to be evaluated on an individual patient basis. Considering the high prevalence of substance abuse and addiction in the United States, involving consultants with addiction medicine expertise on perioperative teams may be a useful approach going forward.

In patients for whom the medication is discontinued preoperatively, it should be stopped three days prior to surgery. As long as the drug has been given sufficient time to be metabolized by the liver and excreted by the biliary system (typically 72 hours), mu-opioid agonists may be effectively used for postoperative analgesia, taking into account that most of these patients are opioid-tolerant and require larger than standard doses. Although the duration of action for buprenorphine is about 40 hours (47), the elimination half-life after a sublingual dose is $37 \mathrm{~h}$ (48). If buprenorphine-naloxone was given within the preceding three days before surgery, its effects may still be present as it has a very strong binding affinity for the $\mu$-opioid receptor and dissociates very slowly from the receptor (47). If opioids are used for postoperative analgesia, the resulting analgesia may not be adequate using routine clinical doses if some of the buprenorphine is still present due to competitive binding of burprenorphine and its "ceiling" effect. Emphasis should be on regional anesthesia techniques and non-opioid agents, especially ketamine as a potent 
analgesic that does not affect respiratory drive (49) and provides the most benefit after painful procedures (50).

For patients who continue taking buprenorphine-naloxone through the perioperative period, buprenorphine can provide sufficient and potent opioid analgesia as a partial mu-opioid agonist, and for minor procedures may be adequate without much supplementation (13). For major surgery, however, additional analgesics will likely be needed. One author recommends the use of short-acting opioids, such as fentanyl, if a once-daily dosing regimen of buprenorphine-naloxone is continued perioperatively (13), though he noted that "much higher" doses might be required. Additional doses of buprenorphine are often sufficient to manage pain within a multimodal analgesic regimen. Trying to "overpower" the effect of buprenorphine with other opioids may provide unpredictable results as well as unpredictable opioid side effects. For upper- or lowerextremity surgery, peripheral nerve blocks can often eliminate the need for supplemental opioids. A multimodal strategy will help minimize opioids. Early involvement by an acute pain management service is recommended.

In the absence of definitive recommendations, the anesthesiologist will help decide whether to discontinue or continue the buprenorphine preoperatively, possibly in conjunction with the prescribing physician who should be involved with post discharge planning and management. It cannot be emphasized enough how important collaboration and coordination are for safe and effective perioperative care of this complicated patient population.

\section{Naltrexone}

In contrast to buprenorphine or methadone, naltrexone is a full opioid competitive antagonist used in the treatment of opioid and alcohol addiction, and is available as a once-daily 
oral agent or a monthly depot injection (Vivitrol). Overcoming the competitive blockade with opioid agonists to achieve analgesia requires markedly high doses of opioid medications and is not recommended. With the plasma half-life of oral naltrexone at $4 \mathrm{~h}$, and that of the active metabolite at $13 \mathrm{~h}$, patients should be advised not to take their once-daily dose on the morning of surgery (13). With the monthly depot injection, the antagonism lasts roughly 30 days, and elective surgery should be delayed until at least 30 days after the last naltrexone injection if possible (13).

Patients treated for opioid addiction benefit from a preoperative plan regarding management of antagonist/mixed agents but may present for surgery, either emergently or having bypassed preoperative assessment, without such a plan. A careful discussion among the patient, the anesthesiologist, and the surgical team is important, as opioids will be less effective or even ineffective in the setting of these agents. A decision will need to be made to proceed, maximizing non-opioid medications in a multimodal strategy, or to delay surgery.

To summarize the recommendations for managing postoperative analgesia for patients taking buprenorphine-naloxone through the perioperative period undergoing moderately or severely painful surgery:

- Incorporate regional anesthesia techniques whenever possible

- Administer non-opioid adjuncts unless contraindicated

- If opioids are utilized, higher doses may be needed, the analgesic effect may be less, and the resulting side effects greater, justifying appropriately increased levels of monitoring

- Consider involving an acute pain management service early 


\section{Acute Pain Management Services in the Perioperative Surgical Home}

The postoperative period presents an excellent opportunity for an anesthesiologist-led acute pain management service (APMS) to actively help guide the patient through hospitalization until discharge and beyond. Delivering the patient safely through the intraoperative period is no longer sufficient. Adequate postoperative analgesia is a key area within the PSH that has an impact on long-term outcomes for the patient. In the case of opioid-tolerant patients, the postoperative period is frequently the most challenging. Careful planning may yield significant improvements for these patients.

Continuous peripheral nerve blocks have continued to increase in popularity, especially in opioid-tolerant patients (51). The APMS has an important role in managing continuous peripheral nerve blocks and providing patient education. At the authors' institution, the APMS physicians and colleagues skilled in regional anesthesia techniques work together to manage these patients who received a peripheral nerve block, including providing 24-hour coverage via a beeper that patients may call for problems or questions. Regional anesthesia and analgesic techniques will likely become even more important as their inclusion in surgical pathways increases (45).

To summarize the benefits of an APMS in the perioperative surgical home in the setting of opioid-tolerant patients in the postoperative period:

- Management of continuous peripheral nerve blocks, including titration, side effects, and ambulatory catheter instructions, from the recovery room until discharge and beyond

- Initiation and management of adjunctive medications, such as ketamine, that require expertise beyond the typical analgesic regimen 
- Guidance and recommendations on opioid conversions and discharge regimens that include high opioid doses

\section{Conclusion}

Management of acute pain with minimization of adverse events in the opioid-tolerant patient undergoing surgery can be extremely challenging. The anesthesiologist, using his or her expertise in regional anesthesia techniques and pharmacology, can improve patient care within the PSH by implementing a comprehensive opioid-reducing strategy for opioid-tolerant patients that begins in the early preoperative period. Intraoperative care of these patients relies heavily on a multimodal analgesic regimen to minimize required opioids, and a postoperative APMS may help transition from the hospital setting to discharge. Anesthesiologists are expected to be involved in the entire perioperative process. Challenging patients, such as the opioid-tolerant, demand our expertise, especially as the specialty of anesthesiology evolves to perioperative medicine.

For those practitioners working in institutions without discrete acute pain services or perioperative surgical home, a good place to start is to initiate discussions with the Quality Assessment and Performance Improvement team to bring these issues to the forefront. Interested practitioners can also approach their anesthesia and surgical colleagues to spark initiatives. Collaboration with anesthesia departments at other institutions who have implemented some elements of the PSH can be extremely helpful, especially with administrative or financial concerns. Changing institutional culture is difficult and individual champions for improvement can be confronted by resentment if they take these issues on alone.

Future study will be needed to demonstrate the efficacy of the PSH, but the benefits seen by similar programs and the support of the ASA give optimism. The Triple Aim requires not only 
anesthesiologist expertise, but also collaboration with physician colleagues and hospital administration. Through continued improvement measures, anesthesiologists are positioned to greatly improve the perioperative continuum. 


\section{References:}

1. Gan TJ, Habib AS, Miller TE, Apfelbaum JL. Incidence, patient satisfaction, and perceptions of post-surgical pain: results from a US national survey. Curr Med Res Opin 2014;30:149-160.

2. Kain Z, Vakharia S, Garson L, et al. The Perioperative Surgical Home as a Future Perioperative Practice Model. Anes Anal, 2014;118(5):1126-1136.

3. Berwick DM, Nolan TW, Whittington J. The triple aim: care, health, and cost. Health Aff, 2008;27:759-69.

4. Vetter TR, Boudreaux AM, Jones KA, et al. The Perioperative Surgical Home: How Anesthesiology Can Collaboratively Achieve and Leverage the Triple Aim in Health Care. Anesth Analg 2014;118(5):1131-1136.

5. Abel R. and Robenblatt A. Preoperative Evaluation and Preparation of Patients for Orthopedic Surgery. Anesthesiology Clin, 2014;32:881-892.

6. Gulur P, Williams L, Chaudhary S, et al. Opioid Tolerance - A Predictor of Increased Length of Stay and Higher Readmission Rates. Pain Phys 2014;17:E503-E507.

7. Institute of Medicine (US) Committee on Advancing Pain Research, Care, and Education. Relieving Pain in America: A Blueprint for Transforming Prevention, Care, Education, and Research. Washington, D.C.: National Academies Press; 2011.

8. Mahathanaruk M, Hitt J, de LeonCasasola OA. Perioperative Management of the Opioid Tolerant Patient for Orthopedic Surgery. Anesthesiology Clin, 2014;32:923-932.

9. Angst M. Intraoperative Use of Remifentanil for TIVA: Postoperative Pain, Acute Tolerance, and Opioid-Induced Hyperalgesia. Journ Cardiothoracic and Vascular Anesthesia, 2015;29(S1):S16-S22. 
10. Kim SH, Stoicea N, Soghomonyan S, et al. Intraoperative Use of Remifentanil and Opioid Induced Hyperalgesia/Acute Opioid Tolerance: Systematic Review. Front Pharmacol, $2014 ; 5: 108$.

11. Lee M, Silverman S, Hansen H, et al. A Comprehensive Review of Opioid-Induced Hyperalgesia. Pain Physician, 2011;14:145-61.

12. Yi P, Pryzbylkowski P. Opioid Induced Hyperalgesia. Pain Medicine 2015;16:S32-S36.

13. Bryson E. The Perioperative Management of Patients Maintained on Medications Used to Manage Opioid Addiction. Curr Opin Anes, 2014;27:359-364.

14. Peng PWH, Tumber PS, Gourlay D. Review article: Perioperative pain management of patients on methadone therapy. Can J Anesth, 2005;52(5):513-523.

15. Bornemann-Cimenti H, Lederer AJ, Wejbora M, et al. Preoperative Pregabalin Administration Significantly Reduces Postoperative Opioid Consumption and Mechanical Hyperalgesia After Transperitoneal Nephrectomy. Br J Anaesth, 2012;108:845-9.

16. Buvanendran A, Kroin JS, Della Valle CJ, et al. Perioperative Oral Pregabalin Reduces Chronic Pain After Total Knee Arthroplasty: A Prospective, Randomized, Controlled Trial. Pain Med ,2010;110(1):199.

17. Sawan H, Chen AF, Viscusi ER, et al. Pregabalin reduces opioid consumption and improves outcome in chronic pain patients undergoing total knee arthroplasty. Phys Sports Med. 2014;42(2):10-18.

18. Kim JC, Choi YS, Kim KN, et al. Effective Dose of Peri-operative Oral Pregabalin as an Adjunct to Multimodal Analgesic Regimen in Lumbar Spinal Fusion Surgery. Spine, 2011;36(6):428-33. 
19. Derry S, Moore RA. Single Dose Oral Celecoxib for Acute Postoperative Pain in Adults. Cochrane Dat Sys Rev, 2013;10:CD004233.

20. Trabulsi EJ, Patel J, Viscusi ER, et al. Preemptive Multimodal Pain Regimen Reduces Opioid Analgesia for Patients Undergoing Robotic-assisted Laparoscopic Radical Nephrectomy. Urology, 2010;76(5):1122-1124.

21. Welchek, C.M., Mastrangelo, L., Sinatra, R.S. et al, Qualitative and quantitative assessment of pain. in: R. Sinatra, O.A. de Leon-Cassasola, B. Ginsberg et al, (Eds.) Acute pain management. Cambridge University Press, New York; 2009:147-166.

22. Larcher A, Laulin JP, Celerier E, Et al. Acute tolerance associated with a single opiate administration: involvement f N-methyl-D-aspartate-dependent pain facilitatory systems. Neuroscience 1998;84(2):583-9.

23. Shimoyama N, Shimoyama M, Inturrisi CE, et al. Ketamine attenuates and reverses morphine tolerance in rodents. Anesthesiology 1996;85:1357-66.

24. Laulin JP, Marette P, Corcuff JB, et al. The role of ketamine in preventing fentanylinduced hyperalgesia and subsequent acute morphine tolerance. Anest Analg 2001;94(5)263-9.

25. Subramaniam K, Subramaniam B, Steinbrook RA. Ketamine as adjuvant analgesic to opioids: A quantitative and qualitative systematic review. Anesth Analg 2004;99(2):48295.

26. Bell RF, Dahl JB, Moore RA, Kalso E. Perioperative ketamine for acute postoperative pain. Cochrane Database Syst Rev 2006;(1):CD004603. 
27. Loftus RW, Yeager MP, Clark JA, et al. Intraoperative ketamine reduces perioperative opiate consumption in opiate-dependent patients with chronic back pain undergoing back surgery. Pain Medicine. 2010;113:639-46.

28. Schwenk ES, Baratta JL, Gandhi K, Viscusi ER. Setting Up an Acute Pain Management Service. Anesthesiol Clin 2014;32:893-910.

29. Ilfeld BM. Continuous peripheral nerve blocks: A review of the published evidence. Anesth Analg 2011;113(4):904-25.

30. Capdevila X, Berthelet Y, Biboulet P, et al. Effects of perioperative analgesic technique on the surgical outcome and duration of rehabilitation after major knee surgery. Anesthesiology 1999;91(1):8-15.

31. McCarthy GC, Megalla SA, Habib AS. Impact of intravenous lidocaine infusion on postoperative analgesia and recovery from surgery: a systematic review of randomized controlled trials. Drugs 2010;70(9):1149-63.

32. Wongyingsinn M, Baldini G, Charlebois P, et al. A randomized controlled trial in patients undergoing laparoscopic colorectal surgery using an enhanced recovery program. Reg Anesth Pain Med 2011;36:241-48.

33. Terkawi AS, Durieux ME, Gottschalk A, Brenin D, Tiouririne M. Effect of intravenous lidocaine on postoperative recovery of patients undergoing a mastectomy: a double-blind, placebo-controlled randomized trial. Reg Anesth Pain Med 2014;39(6):472-7.

34. Poree LR, Guo TZ, Kingery WS, Maze M. The analgesic potency of dexmedetomidine is enhanced after nerve injury: a possible role for peripheral alpha2-adrenoreceptors. Anesth Analg 1998;87:941-8. 
35. Tufanogullari B, White PF, Peixoto MP, Kianpour D, Lacour T, Griffin J, Skrivanek G, Macaluso A, Shah M, Provost DA. Dexmedetomidine infusion during laparoscopic bariatric surgery: the effect on recovery outcome variables. Anesth Analg, 2008;106(6):1741-8.

36. Gurbet A, Basagan-Mogol E, Turker G, Ugun F, Kaya N, Ozcan B. Intraoperative infusion of dexmedetomidine reduces perioperative analgesic requirements. Can J Anesth 2006;53(7):646-52.

37. Joshi GP, Ogunnaike BO. Consequences of inadequate postoperative pain relief and chronic persistent postoperative pain. Anesthesiol Clin North America 2005;23:21-36.

38. Larson DW, Lovely JK, Cima RR, et al. Outcomes after implementation of a multimodal standard care pathway for laparoscopic colorectal surgery. Br J Surg 2014;101:1023-1030.

39. Madani A, Fiore JF, Wang Y, et al. An enhanced recovery pathway reduces duration of stay and complications after open pulmonary lobectomy. Surgery 2015;158:899-910.

40. Michelson JD, Addante RA, Charlson MD. Multimodal Analgesia Therapy Reduces Length of Hospitalization in Patients Undergoing Fusions of the Ankle and Hindfoot. Foot Ankle Int 2013;34:1526-1534.

41. Kehlet H, Jensen TS, Woolf CJ. Persistent postsurgical pain: risk factors and prevention. Lancet 2006;367:1618-25.

42. Hurley RW, Cohen SP, Williams KA, et al. The Analgesic Effects of Perioperative Gabapentin on Postoperative Pain: A Meta-Analysis. Reg Anesth Pain Med 2006;31:23747.

43. Zhang J, Ho KY, Wang Y. Efficacy of pregabalin in acute postoperative pain: a metaanalysis. Br J Anaesth 2011;106:454-62. 
44. Elia N, Lysakowski C, Tramer MR. Does Multimodal Analgesia with Acetaminophen, Nonsteroidal Antiinflammatory Drugs, or Selective Cyclooxygenase-2 Inhibitors and Patient-controlled Analgesia Morphine Offer Advantages over Morphine Alone? Anesthesiology 2005;103:1296-1304.

45. Walters TL, Mariano ER, Clark JD. Perioperative Surgical Home and the Integral Role of Pain Medicine. Pain Med 2015;16:1666-1672.

46. Viscusi ER. Patient-Controlled Drug Delivery for Acute Postoperative Pain Management: A Review of Current and Emerging Technologies. Reg Anesth Pain Med 2008;33:146.

47. Chen KY, Chen L, Mao J. Buprenorphine-Naloxone Therapy in Pain Management. Anesthesiology 2014;1201262-74.

48. Elkader A, Sproule B. Buprenorphine: Clinical Pharmacokinetics in the Treatment of Opioid Dependence. Clin Pharmacokinet 2005;44:661-680.

49. Craven R. Ketamine. Anaesthesia 2007;62:48-53.

50. Laskowski K, Stirling A, McKay WP, Lim HJ. A systematic review of intravenous ketamine for postoperative analgesia. Can J Anesth 2011;58:911-923.

51. Rapp SE, Ready LB, Nessly ML. Acute pain management in patients with prior opioid consumption: A case-controlled retrospective review. Pain 1995;61:195-201. 